\title{
ESTABILIDAD DEL RENDIMIENTO DE GENOTIPOS DE PAPA (Solanum tuberosum L.)
}

\section{STABILITY OF TUBER YIELDS OF POTATO GENOTYPES (Solanum tuberosum L.)}

\author{
Víctor Vásquez A. ${ }^{1}$, Hector A. Cabrera H. ${ }^{2}$, Luis A. Jiménez D. ${ }^{3}$ y Albarino Colunche ${ }^{4}$
}

\begin{abstract}
Resumen
El objetivo de este estudio fue determinar la estabilidad de genotipos experimentales de papa en cuatro caseríos del Distrito La Encañada, provincia y departamento de Cajamarca. Durante las campañas 2005-2006, 2006-2007, 2007-2008 y 2012-2013 se evaluaron ensayos de rendimiento en seis ambientes contrastantes. El material genético estuvo constituido por seis genotipos experimentales provenientes de la Estación Experimental Baños del Inca-INIA Cajamarca. El diseño experimental utilizado fue bloques completos al azar con tres repeticiones, las parcelas experimentales consistieron de cuatro surcos de $3.0 \mathrm{~m}$ de largo, separados a $1.00 \mathrm{~m}$. El análisis de varianza combinado propuesto por Eberhart \& Russell (1966) reveló significancia estadística $(\mathrm{P} \leq 0.01)$ entre ambientes, genotipo y para la interacción genotipo x medio ambiente (localidades). Los parámetros de estabilidad y adaptabilidad identificaron a los genotipos CAJ010.4 y CAJ004.4 como cultivares prometedores en ambientes favorables e inconsistentes, con rendimientos de 31.36 y $26.57 \mathrm{t}^{\circ} \mathrm{ha}^{-1}$. Se identificaron como los mejores ambientes para rendimiento a las localidades de Santa Clotilde-1 y Chaquilpampa, por los valores de sus índices ambientales positivos y rendimientos de $38.72 \mathrm{t}^{\circ} \mathrm{ha}^{-1}$ y $28.03 \mathrm{t}^{\circ} \mathrm{ha}^{-1}$, superiores a la media general.
\end{abstract}

Palabras clave: genotipo, ambiente, interacción, estabilidad, rendimiento.

\begin{abstract}
The objective of this study was to determine the stability of experimental potato genotypes in four villages of La Encañada District, province and department of Cajamarca. During the 20052006, 2006-2007, 2007-2008 and 2012-2013 campaigns, performance trials were evaluated in six contrasting environments. The genetic material consisted of six experimental genotypes from the Baños del Inca Experimental Station-INIA Cajamarca. The experimental design used was complete blocks at random with three repetitions. The experimental plots consisted of four rows of $3.0 \mathrm{~m}$ long, separated at $1.00 \mathrm{~m}$. The combined analysis of variance proposed by Eberhart \& Russell (1966), revealed statistical significance $(\mathrm{P} \leq 0.01)$ between environments, genotype and for the interaction genotype $\mathrm{x}$ environment (localities). The parameters of stability and adaptability identified the genotypes CAJ010.4 and CAJ004.4, as promising cultivars in favorable and inconsistent environments, with yields of 31.36 and $26.57 \mathrm{t}^{\circ} \mathrm{ha}^{-1}$. They were identified as the best environments for yield to the towns of Santa Clotilde and Chaquilpampa, for the values of their positive environmental indices and yields of $38.72 \mathrm{t}^{\circ} \mathrm{ha}^{-1}$ and $28.03 \mathrm{t}^{\circ} \mathrm{ha}^{-1}$, higher than the general average. Key words: genotype, environment, interaction, stability, performance.
\end{abstract}

\section{Introducción}

El mejoramiento genético de la papa (Solanum tuberosum L.) está orientado principalmente a formar cultivares de alto rendimiento comercial y buena estabilidad, aún en presencia de factores bióticos y abióticos adversos, lo que ha permitido satisfacer las demandas de los agricultores, del mercado para consumo fresco y de la agroindustria. La buena calidad del tubérculo está influenciada por factores genéticos, ambientales, de manejo agronómico y por las interrelaciones entre éstos, pero sólo la variabilidad genética y la interacción genotipo x ambiente (IGA) son de importancia para el fitomejorador, por lo que es necesario determinar su contribución en el comportamiento del fenotipo (Vásquez, 1988; Milton \& Allen, 1995).

La interacción GA entendida como un cambio del comportamiento relativo de los genotipos a través de ambientes cambiantes, causa confusión en la estimación de parámetros genéticos reduciendo la respuesta a la selección y dificulta la identificación de genotipos superiores. Su análisis e interpretación permite identificar mega-ambientes al detectar genotipos estables en un grupo de ambientes diferentes 
o con mayor rendimiento en uno de éstos, propone estrategias de mejoramiento genético y genera tecnología que permita a los agricultores elegir la mejor variedad en una región (Kang, 2002).

Para el estudio de la interacción genotipo ambiente (IGA) y la estabilidad se han empleado varios modelos estadísticos, entre los cuales, el de Eberhart \& Russell (1966) propone que un genotipo es estable si: a) $\mathrm{Su}$ varianza entre ambientes es pequeña, a este grupo pertenece $S^{2}{ }_{d}$ y b) Su respuesta al ambiente es paralela a la respuesta promedio de todos los genotipos evaluados, a este grupo pertenecen $\beta_{\mathrm{i}}$.

En la Región Cajamarca, predominan condiciones ambientales heterogéneas entre localidades, entre años o entre las combinaciones de éstos, por lo que la identificación de genotipos de alto rendimiento y estabilidad podría ser una alternativa de solución para estas áreas. Existe poca información en la literatura especializada sobre el potencial de rendimiento y su estabilidad de los cultivares utilizados por los agricultores. Con base a lo mencionado anteriormente, en este experimento se compararon 6 genotipos en seis ambientes diferentes para identificar aquellos de mayor rendimiento y estabilidad mediante el modelo de Eberhart \& Russell (1966).

\section{Metodología}

Seis genotipos avanzados de papa, se evaluaron en 6 experimentos durante la campaña bajo secano, de septiembre a octubre de 2007 al 2012 (Tabla 1). Los datos climáticos predominantes en las localidades se presentan en la Tabla 2. La unidad experimental consistió de 4 surcos de $1.0 \mathrm{~m}$ de ancho por $3.0 \mathrm{~m}$ de largo, y los dos surcos centrales se usaron como unidad experimental útil, de 10 plantas dispuestas a $0.30 \mathrm{~m}$ entre sí $\left(6.00 \mathrm{~m}^{2}\right)$.

Variables de respuesta

Al finalizar el ciclo del cultivo, se registró el peso total de tubérculos y el peso comercial en $\mathrm{kg} /$ parcela.

\section{Diseño experimental}

Para la ejecución en campo se utilizó el diseño de bloques completos al azar (DBCA), con tres repeticiones, de acuerdo al siguiente modelo matemático:

$$
y_{i j l}=\mu+G_{i}+B_{j(l)}+A_{l}+(G A)_{i l}+\varepsilon_{i j l}
$$

Donde:

$\mathrm{Y}=$ Valor del carácter estudiado; $\mathrm{A}=$ Efecto de ambiente; $\mu=$ Media general; $(\mathrm{G} \mathrm{A})_{\mathrm{il}}=$ Efecto de la interacción genotipo ambiente; $\mathrm{G}=$ Efecto de genotipo; $\varepsilon_{\mathrm{ijl}}=$ Error experimental; $\mathrm{B} \mathrm{j}(\mathrm{l})=$ Efecto de bloques dentro de ambientes.

Tabla 1. Genotipos utilizados en el experimento.

\begin{tabular}{ll}
\hline Genotipos & Pedigree \\
\hline Canchán & BL-1.2 x Murillo III-80 \\
CAJ004.4 & 392657.8 x E (18) x BulkB \\
CAJ010.5 & 61 T.5 x Bulk1 (4) BulkA \\
CAJ003.4 & 61 T.5 x Bulk1 (1) BulkA \\
CAJ010.1 & 61T.5 x Bulk1 (4) BulkA \\
CAJ010.4 & $61 T .5 \times$ Bulk1 (4) BulkA \\
\hline
\end{tabular}

\section{Análisis estadístico}

Los genotipos se dispusieron en el campo en un diseño experimental de bloques completos al azar con 3 repeticiones. Se realizaron análisis de varianza por localidad y año. Se utilizó la prueba de Bartlett (Vásquez, 2014) para determinar homogeneidad de varianzas de los errores experimentales en cada localidad. En vista de que se encontró homogeneidad en las varianzas de los errores experimentales en la totalidad de las variables de respuesta, se decidió realizar el análisis de varianza combinado.

Se efectuaron pruebas de comparación de medias de Duncan al 0.05 de probabilidad.

El análisis combinado se realizó principalmente para identificar la existencia o no de la interacción genotipo $\mathrm{x}$ ambiente. Esta fuente de variación permitirá determinar la estabilidad fenotípica de los genotipos a través de las 6 localidades mediante el modelo propuesto por Eberhart \& Russell (1966). Los datos se analizaron siguiendo un arreglo de tratamientos en diseño de bloques al azar. Las localidades y los genotipos se consideraron como efectos fijos.

Una vez que se ha encontrado una interacción genotipo por localidad (ambiente) en las variables de respuesta, se recurrió al análisis de estabilidad propuesto por Eberhart \& Russell (1966), para analizar

Tabla 2. Índices climáticos de la Encañada durante ocho años que se presentaron durante el desarrollo del cultivo (2005-2013).

\begin{tabular}{lccccc}
\hline Años & \multicolumn{2}{c}{ Temperatura $\left(\mathrm{C}^{\circ}\right)$} & Precipitación & $\begin{array}{c}\text { Humedad } \\
(\mathrm{mm})\end{array}$ & $(\%)$ \\
\hline $2005-2006$ & 18.97 & 7.84 & 13.41 & 94.00 & 67.00 \\
$2006-2007$ & 18.69 & 7.75 & 13.22 & 66.40 & 65.00 \\
$2007-2008$ & 18.55 & 7.50 & 13.03 & 96.80 & 70.00 \\
$2008-2009$ & 17.33 & 7.14 & 12.24 & 153.60 & 88.00 \\
$2009-2010$ & 18.38 & 7.60 & 12.99 & 140.48 & 84.00 \\
$2010-2011$ & 18.78 & 7.53 & 13.16 & 52.00 & 72.00 \\
$2011-2012$ & 19.30 & 7.90 & 13.60 & 97.36 & 80.00 \\
$2012-2013$ & 19.90 & 8.62 & 14.26 & 80.00 & 75.00 \\
Promedio & 18.73 & 7.73 & 13.23 & 97.58 & 75.12 \\
\hline
\end{tabular}


la interacción, primordialmente respecto al rendimiento de tubérculo comercial.

Este modelo estadístico nos permite estimar los parámetros de estabilidad para cada genotipo a partir del siguiente modelo de regresión lineal:

$$
Y_{i j}=\mu_{i}+\beta_{i} I_{j}+\delta_{i j}
$$

Donde:

Yij es la media del genotipo i-ésimo en el j-ésimo ambiente; $\mu_{i}$ corresponde a la media del i-ésimo genotipo sobre todos los ambientes; $\beta_{i}$ es el coeficiente de regresión que mide la respuesta del i-ésimo genotipo en varios ambientes; $\mathrm{I}_{\mathrm{j}}$ corresponde al índice ambiental que se obtiene a partir de la diferencia de la media del j-ésimo ambiente con la correspondiente media general; $\delta_{i j}$ es la desviación de regresión lineal del iésimo genotipo en el j-ésimo ambiente.

Para cada genotipo se realizó un análisis de regresión, utilizando el índice ambiental como la variable independiente y el rendimiento comercial de tubérculo como variable dependiente. De este modo, el efecto del ambiente puede ser descompuesto en 2 componentes, uno lineal y su residuo. El coeficiente de regresión $\beta$ i está asociado con el componente lineal, indicando la adaptación del genotipo, o bien, su capacidad de respuesta entre los distintos ambientes. Los desvíos de la regresión $\left(\delta_{i j}\right.$ ) están asociados al componente no lineal e indican estabilidad genotípica. Un genotipo con un $\delta_{i j}=0$, muestra un comportamiento predecible de acuerdo con la magnitud del índice ambiental.

Para definir la estabilidad y consistencia de los genotipos de papa se utilizó la clasificación propuesta por Carballo \& Márquez (1970) (Tabla 3). Esta información señala que un coeficiente de regresión de $\mathrm{b}=1$ corresponde a un genotipo estable, en tanto que valores mayores $(b>1)$ corresponden a genotipos que responden mejor a ambientes buenos, y valores menores $(b<1)$ indican a los materiales que funcionan mejor en ambientes desfavorables. Respecto a las desviaciones de regresión, un valor $S_{d_{i}}^{2}=0$ indica una variedad estable, en tanto que valores mayores $\left(S_{d_{i}}^{2}>\right.$ $0)$ corresponden a materiales inconsistentes.

La hipótesis de que cualquier coeficiente de regresión no fuese distinto a la unidad se evaluó mediante una prueba de "t"; mientras que la hipótesis de que los desvíos de la regresión no fueran diferentes de 0 se verificó mediante la prueba de F.
Tabla 3. Situaciones posibles derivadas de los valores de los parámetros de estabilidad (Carballo \& Márquez 1970).

\begin{tabular}{cccl}
\hline E & $\begin{array}{c}\text { Coeficiente } \\
\text { de } \\
\text { regresión } \\
\text { (bi) }\end{array}$ & $\begin{array}{c}\text { Desviación } \\
\text { de regresión } \\
\left(S_{d_{i}}^{2}\right)\end{array}$ & \multicolumn{1}{c}{ Descripción } \\
\hline A & 1.0 & $S_{d_{i}}^{2}=0$ & Variedad estable \\
B & 1.0 & $S_{d_{i}}^{2}>0$ & $\begin{array}{l}\text { Buena respuesta en } \\
\text { todos los ambientes, } \\
\text { inconsistente. }\end{array}$ \\
C & $<1$ & $S_{d_{i}}^{2}=0$ & $\begin{array}{l}\text { Buena en ambientes } \\
\text { desfavorables, } \\
\text { consistente. }\end{array}$ \\
D & $<1$ & $S_{d_{i}}^{2}>0$ & $\begin{array}{l}\text { Buena en ambientes } \\
\text { desfavorables, } \\
\text { inconsistente. }\end{array}$ \\
& & & $\begin{array}{l}\text { Buena en ambientes } \\
\text { favorables, } \\
\text { consistente. }\end{array}$ \\
E & $>1$ & $S_{d_{i}}^{2}=0$ & $\begin{array}{l}\text { Buena en ambientes } \\
\text { favorables, } \\
\text { inconsistente. }\end{array}$ \\
F & $>1$ & $S_{d_{i}}^{2}>0$ &
\end{tabular}

\section{Resultados \\ Análisis de variabilidad}

En los análisis de varianza individuales se encontraron diferencias altamente significativas entre genotipos $(\alpha=0.01)$ para la variable registrada. La prueba de Bartlett (Vásquez, 2014), indicó que las varianzas fueron homogéneas. El análisis conjunto arrojó diferencias altamente significativas entre localidades (ambientes) y entre genotipos (Tabla 4). De acuerdo con estos resultados los genotipos evaluados se comportaron de forma diferente a través de los seis ambientes evaluados.

Interacción genotipo por ambiente

La Interacción Genotipo x Ambiente (IGA) resultó altamente significativa $(\mathrm{p}<0.01)$ para la variable rendimiento de tubérculo comercial. Estos resultados demuestran que los genotipos tienen un comportamiento desigual en los diferentes ambientes probados, esto es debido a la gran influencia que ejerce el ambiente sobre el rendimiento (Tabla 4). El coeficiente de variación (5.72\%) es bajo, lo que sugiere, de acuerdo con Reyes (1990) y Vásquez (2014), que la conducción de los experimentos y los resultados obtenidos son confiables.

Efecto de localidades

En el presente experimento las localidades capturaron el $46.71 \%$ de la suma de cuadrados total del análisis de varianza, para el rendimiento de tubérculo comercial. Esto indica que los ambientes fueron 
Tabla 4. Análisis de varianza combinado de 6 genotipos avanzados de papa en 6 localidades diferentes para rendimiento comercial.

\begin{tabular}{lrrrrl}
\hline Fuente de variación & Grados de libertad & Suma de cuadrados & Cuadrado medio & F $_{\text {Calculado }}$ & Probabilidad \\
\hline Localidades (Loc) & 5 & 1680.56 & 336.11 & $445.49 * *$ & $<0.0001$ \\
Rep/loc & 12 & 3.27 & 0.27 & 0.36 & 0.2650 \\
Genotipos & 5 & 961.21 & 192.24 & $254.80^{* *}$ & $<0.0001$ \\
Genotipos x Loc & 25 & 884.77 & 35.39 & $46.91^{* *}$ & $<0.0001$ \\
Error combinado & 60 & 45.27 & 0.755 & & \\
Total & 107 & 3577.07 & & & \\
\hline
\end{tabular}

** Significación estadística a 0.01 de probabilidad de error. $\mathrm{R}^{2}=0.987, \quad \mathrm{CV}=5.72 \%, \bar{x}=15.17083$.

diferentes y contrastantes, con diferencias en la magnitud de las respuestas entre las medias ambientales. El rendimiento de tubérculo comercial y la prueba de Duncan $(\alpha=0.05)$ se presentan en la Tabla 5. Mediante el modelo de Eberhart \& Russell (1966) se identificaron como los mejores ambientes para rendimiento a: Sta Clotilde-1 y Chaquilpampa, por los valores de sus índices ambientales positivos, es decir, superiores a la media general; y los ambientes con mal comportamiento fueron: Sta Margarita-6, Sta Clotilde2, Sta Margarita-5 y Sta Margarita-4, respectivamente, por tener índices ambientales negativos, es decir, menores a la media general.

Tabla 5. Prueba de comparación múltiple de Duncan para localidades al $5 \%$ para rendimiento de tubérculo comercial de papa.

\begin{tabular}{llc}
\hline Localidades & ${\mathrm{t} \bullet h \mathrm{a}^{-1}}^{-1}$ & Índice Ambiental \\
\hline Sta Clotilde -1 & $38.72 \mathrm{a}$ & 8.0171 \\
Chaquilpampa & $28.03 \mathrm{~b}$ & 1.6568 \\
Sta Margarita-6 & $22.63 \mathrm{c}$ & -1.5829 \\
Sta Clotilde -2 & $21.99 \mathrm{c}$ & -1.9690 \\
Sta Margarita-5 & $20.89 \mathrm{~d}$ & -2.6329 \\
Sta Margarita-4 & $19.64 \mathrm{e}$ & -3.4884
\end{tabular}

Efecto de genotipos

El análisis de varianza para genotipos mostró diferencias altamente significativas entre los distintos genotipos avanzados de papa evaluados para el rendimiento de tubérculo comercial; se logró capturar el $26.81 \%$ de la suma de cuadrados total del análisis de varianza de esta variable.

En la Tabla 6 se muestran los rendimientos promedios por parcela y toneladas por hectárea $\left(\mathrm{t} \bullet \mathrm{ha}^{-1}\right)$ de tubérculo comercial de los seis genotipos de papa. Las medias de los genotipos variaron desde 32.29

Tabla 6. Prueba de Duncan $(\alpha=0.05)$ para el rendimiento de seis genotipos de papa evaluados en seis localidades.

\begin{tabular}{lc}
\hline Genotipos & Rendimiento t•ha ${ }^{1}$ \\
\hline CAJ010.4 & $32.29 \mathrm{a}$ \\
CAJ004.4 & $27.82 \mathrm{a} \mathrm{b}$ \\
CAJ003.4 & $26.58 \mathrm{a} \mathrm{b}$ \\
CAJ010.1 & $25.80 \mathrm{a} \mathrm{b}$ \\
CAJ010.5 & $23.42 \mathrm{~b}$ \\
CANCHAN & $15.97 \mathrm{c}$ \\
\hline
\end{tabular}

$\mathrm{t} \bullet \mathrm{ha}^{-1}$ en el genotipo CAJ10.4, hasta $15.97 \mathrm{t}^{\circ} \mathrm{ha}^{-1}$ en el genotipo CANCHAN, casi una relación 2.1 veces mayor de la primera respecto a la última. La prueba de Duncan indica que no hay diferencias estadísticas significativas entre los cuatro primeros genotipos, destacando el genotipo CAJ010.4 con $32.29 \mathrm{t}^{\bullet} \mathrm{ha}^{-1}$ frente a los genotipos CAJ010.5 y CANCHAN que ocupan los últimos lugares con 23.42 y $15.97 \quad$ t•ha-1, respectivamente.

Análisis de estabilidad

Una vez evidenciada la presencia de la interacción G x A se analizó la estabilidad de rendimiento por el método de Eberhart \& Russell (1966), del rendimiento (Tabla 7), en el cual se aprecia significación estadística entre genotipos. Esto indica que su comportamiento promedio en todos los ambientes fue muy variado. El comportamiento desigual de los genotipos justifica la utilización de los parámetros de estabilidad. La interacción genotipo $\mathrm{x}$ ambiente, al no ser significativa indica que no hubo respuesta diferencial del rendimiento de los genotipos a través de los diferentes ambientes de prueba.

La información presentada en la Tabla 8 permite analizar el rendimiento medio de cada genotipo, la prueba de rango múltiple de Duncan, y los valores estimados de los parámetros de estabilidad como es el coeficiente de regresión $\left(b_{i}\right)$, las desviaciones de regresión $\left(s_{d i}^{2}\right)$ y la significancia estadística de estos parámetros. También se señala la caracterización de cada genotipo en cuanto a su comportamiento en buenos y malos ambientes, así como la consistencia de cada genotipo.

Atendiendo al criterio de Carballo \& Márquez (1970), para definir la estabilidad de un genotipo (bi = $1 \mathrm{y} s_{d i}^{2}=0$ ), se puede afirmar que los genotipos CAJ003.4 y CAJ010.5 mostraron un coeficiente de regresión igual a 1.0, de modo que tuvieron la mayor estabilidad y mayor rendimiento.

El parámetro de las desviaciones de la regresión $\left(s_{d i}^{2}\right)$ para los tres genotipos antes mencionados resultó significativamente diferente de 0 . Consecuentemente, estos genotipos también deben considerarse inconsistentes. 
Tabla 7. Significancias de cuadrados medios para estimar los parámetros de estabilidad en 6 genotipos avanzados de papa (Solanum tubersosum L.) en Cajamarca, Perú.

\begin{tabular}{|c|c|c|c|}
\hline $\begin{array}{l}\text { Fuente de } \\
\text { variación }\end{array}$ & $\begin{array}{l}\text { Grados de } \\
\text { libertad }\end{array}$ & $\begin{array}{l}\text { Cuadrado } \\
\text { medio }\end{array}$ & $\mathrm{F}_{\text {calculado }}$ \\
\hline Total & 35 & & \\
\hline Genotipos & 5 & 63.502619 & $5.9912 * *$ \\
\hline $\begin{array}{l}\text { Ambiente }+(G \\
\text { x Amb })\end{array}$ & 30 & 26.830000 & $2.5200 *$ \\
\hline $\begin{array}{l}\text { Ambiente } \\
\text { (Lineal) }\end{array}$ & 1 & & \\
\hline $\mathrm{G} \quad \mathrm{x}$ & 5 & 7.666900 & 0.7233 \\
\hline Amb.(Lineal) & & & \\
\hline $\begin{array}{l}\text { Desviación } \\
\text { conjunta }\end{array}$ & 24 & 10.59936 & \\
\hline CANCHAN & 4 & 25.73509 & $34.1098 * *$ \\
\hline CAJ004.4 & 4 & 4.72678 & $6.2650 * *$ \\
\hline CAJ010.5 & 4 & 3.74225 & $4.9601 * *$ \\
\hline CAJ003.4 & 4 & 4.61622 & $6.1184 * *$ \\
\hline CAJ010.1 & 4 & 16.58137 & $21.9773 * *$ \\
\hline CAJ010.4 & 4 & 7.87640 & $10.4395 * *$ \\
\hline Error conjunto & 60 & 0.754477 & \\
\hline
\end{tabular}

En la misma Tabla 8 se observa que los genotipos CAJ010.4 y CAJ004.4 presentan un coeficiente de regresión mayor que 1 (bi >1) considerándose como buenos en ambientes favorables. La significación de las desviaciones de regresión $\left(s_{d i}^{2}\right)$ para dichos genotipos resulta ser significativamente mayor que cero, considerándose como inconsistentes.

El genotipo CAJ010.1 presenta un coeficiente de regresión menor que 1 y una desviación de regresión mayor que cero $\left(s_{d i}^{2}>0\right)$. Este genotipo es bueno en
Tabla 8. Rendimiento promedio de tubérculo comercial y parámetros de estabilidad de 6 genotipos avanzados de papa evaluados en 6 ambientes de Cajamarca.

\begin{tabular}{llcc}
\hline Genotipo & $\begin{array}{c}\text { Rendimiento } \\
\mathrm{t} \cdot \mathrm{ha}^{-1}\end{array}$ & $\begin{array}{c}\text { Coeficiente } \\
\text { de regresión } \\
\left(\boldsymbol{b}_{\boldsymbol{i}}\right)\end{array}$ & $\left.\begin{array}{c}\text { Desviaciones } \\
\text { de regresión } \\
\left(s_{d i}^{2}\right.\end{array}\right)$ \\
\hline CAJ010.4 & $31.36 \mathrm{a}$ & $1.40 *$ & $7.78 * *$ \\
CAJ004.4 & $26.57 \mathrm{ab}$ & $1.24 * *$ & 1.68 \\
CAJ003.4 & $26.30 \mathrm{ab}$ & $0.82 *$ & $\mathbf{3 . 5 9} * *$ \\
CAJ010.1 & $25.30 \mathrm{ab}$ & 0.65 & $13.85 * *$ \\
CAJ010.5 & $22.13 \mathrm{~b}$ & $1.03 * *$ & $\mathbf{1 . 4 9}$ \\
CANCHAN & $14.82 \mathrm{c}$ & 0.82 & $\mathbf{2 5 . 4 8 * *}$ \\
\hline
\end{tabular}

$$
\bar{x}=25.28 \text {. }
$$

ambientes desfavorables e inconsistente (Carballo \& Márquez 1970).

Por su parte, en la Figura 1, el genotipo CAJ010.5, ubicado en el cuadrante I, además de presentar un rendimiento de tubérculo comercial inferior a la media general, muestra una mayor respuesta del rendimiento con el cambio ambiental (bi >1). Junto con los genotipos CAJ010.1, CAJ003.4, CAJ004.4 y CAJ010.4, aquellos que se encuentran en los cuadrantes II y III, presentaron un rendimiento de tubérculo comercial superior a la media general. En el primer caso, el rendimiento de tubérculo comercial de los clones CAJ004.4 y CAJ010.4, se incrementó más que el correspondiente al promedio de todos los materiales (bi >1). Mientras que en el segundo, el rendimiento total de tubérculo comercial de los genotipos CAJ003.4 y CAJ010.1 (Cuadrante III) se caracterizó por responder menos que el promedio de la

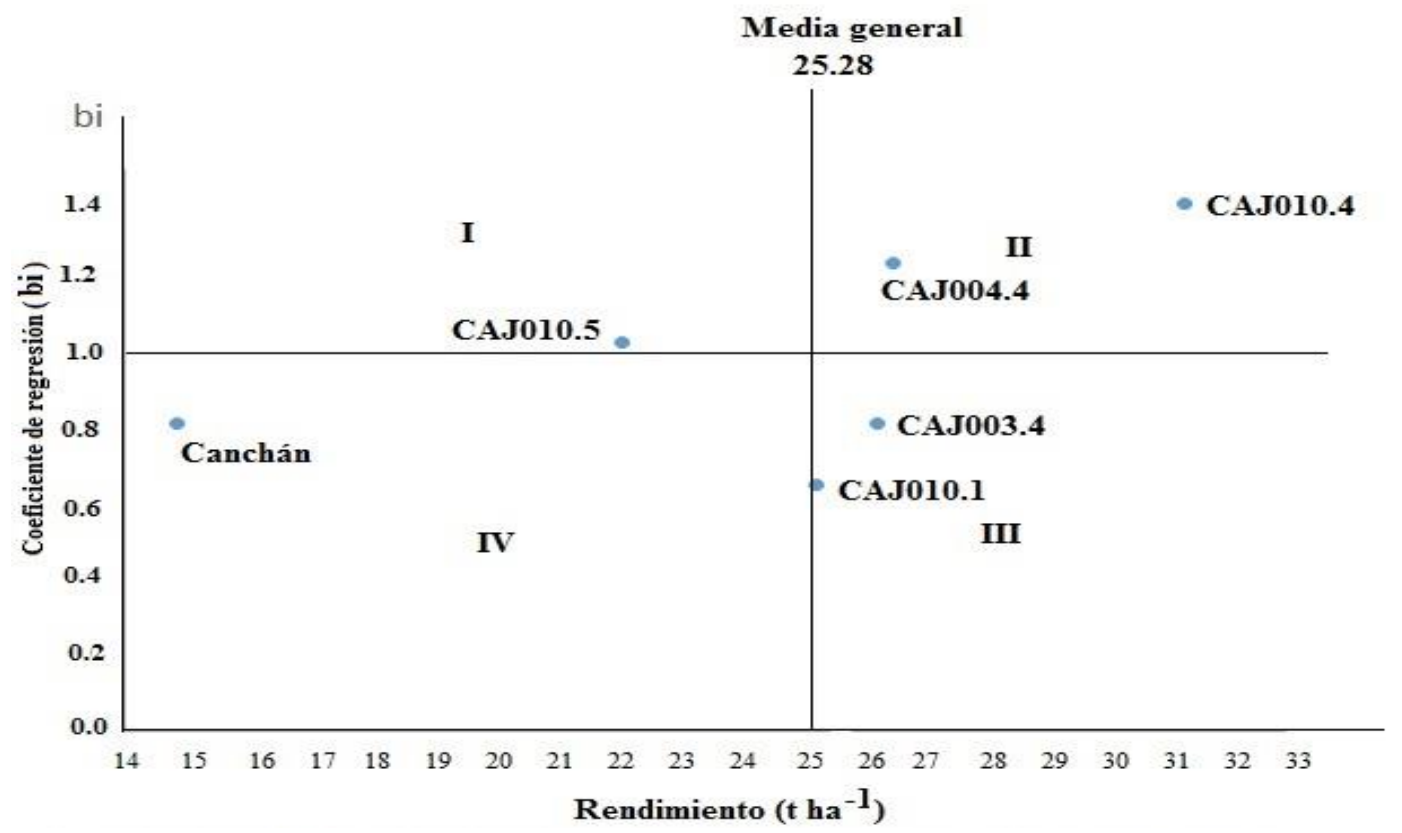

Figura 1. Relación entre la media de rendimiento de tubérculo comercial de seis genotipos de papa con sus respectivos coeficientes (bi) y la media general. 
totalidad de variedades en respuesta al cambio ambiental (del menos al más favorable) (b <1). En resumen, el análisis de estabilidad permitió la identificación de los genotipos CAJ003.4 y CAJ004.4 como los más recomendables para las 6 localidades, mientras que el genotipo CAJ010.1 podría recomendarse únicamente para los ambientes más desfavorables de producción; y el genotipo CAJ010.4 para los más favorables.

\section{Discusión}

Análisis de variabilidad

El análisis combinado para la variable rendimiento comercial de tubérculo mostró alta significancia estadística para Ambientes, Genotipos y su respectiva interacción genotipos por ambiente (Tabla 4).

Interacción genotipo por ambiente

La interacción genotipo por ambiente (IGA) es una característica que se evalúa en los genotipos sobresalientes con el propósito de seleccionar aquellos con una menor interacción en la región de interés, y cuya respuesta en rendimiento se incremente conforme mejoran las condiciones del cultivo (Vásquez, 1988; Crossa et al., 1990; Vásquez, 2014;). Cabe destacar que el rendimiento es un carácter cuantitativo gobernado por muchos genes (carácter poligénico) por lo tanto está fuertemente influenciado por el medio ambiente (Vásquez, 1988). Estos resultados justifican la realización del análisis respectivo de la estabilidad (Finlay \& Willkinson, 1963; Eberhart \& Russell, 1966). Se sabe que la IGA, es uno de los problemas para el fitomejorador puesto que la presencia de ésta limita la selección de los mejores genotipos que puedan ser recomendados para todas las localidades evaluadas. (Allar, 1967; Busey, 1983; Vásquez, 1988; Crossa et al., 1990; Kang, 1998; Rea \& Vieira, 2000). Nuestros resultados coinciden con los comunicados por Márquez (1992), Sahagún (1992), Gonzáles et al. (2003), Pérez et al. (2007), García et al. (2010) y Pérez et al. (2010), quienes subrayaron que la IGA significativa causa confusión en la estimación de parámetros genéticos, reduce el progreso de la selección y limita la posibilidad de elegir genotipos superiores.

Análisis de estabilidad

Los clones CAJ010.4, CAJ004.4 y CA010.5 se clasificaron con buena respuesta en todos los ambientes pero inconsistente, una situación que se esperaba ya que estos genotipos obtuvieron rendimientos superiores al rendimiento de la Región Cajamarca (11.08 t•ha $\left.{ }^{-1}\right)$. Estos genotipos de papa darán mayor seguridad a los agricultores porque se comportan mejor en ambientes pobres.

Por el método de Eberhart \& Russell (1966) los genotipos CAJ010.4, CAJ004.4 y CA010.5 presentan adaptación específica a ambientes favorable (bi > 1). Los genotipos CAJ003.4, CAJ10.1 y Canchán presentan adaptación específica a ambientes desfavorable (bi < 1). El genotipo CAJ010.4 fue el que presentó mayor producción media de rendimiento de tubérculo comercial $\left(31.36 \mathrm{t} \bullet \mathrm{ha}^{-1}\right)$, por lo tanto es el más apropiado para ambientes favorables. Por otro lado, el genotipo CAJ003.4 con una producción media de 26.30 t $h^{-1}$, fue el que presenta mayor adaptabilidad a ambientes desfavorables ( $\mathrm{bi}=0.82 *$ ), coincidiendo con lo establecido por Eberhart \& Russell (1966) y Carballo \& Márquez (1970).

La estabilidad, verificada por los desvíos de regresión $\left(s_{d i}^{2}\right)$, indicó que los genotipos CAJ004.4 y CAJ010.5 (no significativo) se comportan como estables. Los genotipos restantes (significativos al 1\%) presentan inestabilidad, según Carballo \& Márquez (1970), Eberhart \& Russell (1966), Vásquez (2013). Los genotipos CAJ010.1 y Canchán mostraron mayor inestabilidad por poseer el desvió estándar $\left(s_{d i}^{2}\right)$ de mayor magnitud (13.8 y 25.48).

El genotipo ideal, es entonces, aquel que presenta alta producción, coeficiente de regresión igual a uno (estabilidad alta) y desvío de la regresión estadísticamente igual a cero (amplia adaptabilidad). Ningún genotipo de papa presentó estas tres características simultáneamente. Sin embargo, el genotipo CAJ010.4, fue el que presentó mayor rendimiento promedio con respecto al promedio de la región y por lo tanto podría ser el genotipo ideal para la selección. Estos resultados son concordantes con los obtenidos por Vásquez (2013) y Gonzáles et al. (2015).

\section{Conclusiones}

Los ambientes con mejor comportamiento de los genotipos en el rendimiento fueron, Santa Clotilde-1 y Chaquilpampa cuyos rendimientos son $38.72 \mathrm{t} \cdot \mathrm{ha}^{-1} \mathrm{y}$ $28.03 \mathrm{t} \cdot \mathrm{ha}^{-1}$.

Se detectaron efectos significativos $(P \leq 0.01)$ para ambientes (A), genotipos (G) e interacción $\mathrm{G} \times \mathrm{A}$.

Los genotipos CAJ010.4 y CAJ004.4 mostraron los mayores rendimientos.

Los parámetros de estabilidad y adaptabilidad identificaron a los genotipos CAJ010.4 y CAJ004.4, como cultivares prometedores en ambientes favorables e inconsistentes.

El genotipo CAJ003.4 presenta una adaptación general a todos los ambientes. Inconsistente.

\section{Literatura citada}

Allar R. 1967. Principios de la mejora genética de las plantas. Omega, Barcelona.

Busey P. 1983. Management of Crop Breeding. p. 3154 in: Wood, D. R. (ed.) Crop Breeding, Foundations of Modern Crop Science Series, Crop Science Society of America, Madison, WI.

Carballo C. \& Márquez S. 1970. Comparación de variedades de maíz del bajío y la Mesa central por su rendimiento y estabilidad. Agrociencia 5(1): 129-146. 
Crossa J., Gauch H.G. \& Zobel R.W. 1990. Additive main effects and multiplicative interaction analysis of two international maize cultivar trials. In: Crop Science, 30: 493-500.

Eberhart S. \& Russell W. 1966. Stability parameters for comparing varieties. Crop Science 6: 36-40.

Finlay K. \& Wilkinson G. 1963. The analysis of adaptation in a plant-breeding programme. Australian Journal of Agriculture Research 14: 742-754.

García R., Magne J., Angulo A., Mamani P., Almanza J., Herbas J., Álvarez V., Navia O. \& Vallejos J. 2010. Interacción genotipo-ambiente y selección participativa de clones promisorios de papa resistentes a tizón, en cuatro zonas paperas de Cochabamba, Bolivia. Fundación PROINPA. Cochabamba, Bolivia.

Gonzáles E., Estévez A., Castillo J., Salomón L., Varela M., Ortiz U. \& Ortiz E. 2003. Análisis de la estabilidad genotípica en el cultivo de la papa (Solanum tuberosum L.) mediante las representaciones biplots. Cultivos Tropicales, vol. 24, no. 1, p. 81-84.

Gonzáles G., Guerrero M., Anchondo N., Sáenz S., \& Magaña, M. 2015. Estabilidad del rendimiento de chile tipo chilaca. Tecnociencia Chihuahua. Vol. IX. Núm. 1. Enero-Abril.

Gonzáles V. 2004. Estabilidad de producción comercial y agruamiento de clones avanzados de papa en el norteeste de México. Tesis para optar el Grado de maestro en Ceincias en Fitomejoramiento. Universidad Autónoma Agraria Antonio Narro. México.

Kang M. 1998. Using genotype by environment interaction for crop cultivar development. Adv. Agron., 62: 199-252.
Kang M. 2002. Genotype-environment interaction: progress and prospects. In: Kang M.S. (ed.) Quantitative Genetics, Genomies and Plant Breeding. 221-243. CABI Publishing.

Márquez S. 1992. La interacción genético-ambiental en genotecnia vegetal. In: memorias del simposio Interacción Genotipo-Ambiente en Genotecnia Vegetal. 26 y 27 de marzo, Sociedad Mexicana de Fitogenética, Guadalajara, Jalisco, p. 1-27.

Milton P. \& Allen S. 1995 Breeding Field Crops. Iowa State University Press, Ames, Iowa, USA. 494 p.

Pérez L., Hurtado G., Franco M., Rivera P., Sahagún C., Balbuena M., Rubí A. \& Gutiérrez R. 2010. Variabilidad genética, diversidad fenotípica e identificación de genotipos sobresalientes de papa. Revista Mexicana de Ciencias Agrícolas, 1(4): 579-592.

Pérez L., Vázquez G., Sahagún C., Rivera P., 2007. Variabilidad y caracterización de diez variedades de papa en tres localidades del estado de México. Revista Chapingo Serie Horticultura 13(1): 13-19, 2007.

Rea R. \& Vieira O. 2000. Interacción genotipo x ambiente y análisis de estabilidad en ensayos regionales de caña de azúcar en Venezuela. Caña de Azúcar 19: 3-15.

Reyes C. 1990. Diseños de experimentos aplicados. Ed. Trillas 3ra.Ed. México. 348 p.

Sahagún C. 1992. El ambiente, el genotipo y su interacción. Rev. Chapingo 79 y 80:5-12.

Vásquez A. 1988. Mejoramiento genético de la papa. 1a. ed. Edit. Amaru Editores, Lima, Perú.

Vásquez A. 2013. Experimentación Agrícola. Soluciones con SAS. 2a. ed. Edita CONCYTEC-FONDECYT, LimaPerú.

Vásquez A. 2014. Diseños experimentales con SAS. Edita CONCYTEC-FONDECYT, Lima-Perú.

\footnotetext{
${ }^{1}$ Departamento de Agronomía, Facultad de Ciencias Agrarias, Universidad Nacional de Cajamarca. Av. Atahualpa $\mathrm{N}^{\circ}$ 1050. vvarce@ hotmail.com.

2 INIA, BAÑNOS DEL INCA, CAJAMARCA. hcabrera@inia.gob.pe.

${ }^{3}$ Facultad de Economía y Planificación, Universidad Nacional Agraria La Molina. Av. La Molina s/n, Lima 12, Perú.jdl@lamolina.edu.pe.

${ }^{4}$ Departamento de Agronomía, Facultad de Ciencias Agrarias, Universidad Nacional de Cajamarca. Av. Atahualpa N¹050. Alva5129@hotmail.com.
} 